\title{
Could ruxolitinib be effective in patients with COVID-19 infection at risk of acute respiratory distress syndrome (ARDS)?
}

\author{
Giovanni Caocci ${ }^{1}$ (B) $\cdot$ Giorgio La Nasa ${ }^{1}$
}

Received: 31 March 2020 / Accepted: 27 April 2020 / Published online: 14 May 2020

(C) Springer-Verlag GmbH Germany, part of Springer Nature 2020

Dear Editor,

As of March 29, the COVID-19 pandemic has infected around 670,000 people worldwide, causing over 30,000 deaths. Italy was among the countries in the world severely affected by the pandemic with around 80,000 infected people and over 10,000 deaths [1]. The lethality from COVID-19 infection varies from 2 to $9 \%$ and is particularly high, up to $15 \%$, in elderly patients over 65 years of age. The current therapeutic strategies to deal with infection are exclusively supportive and acute respiratory distress syndrome (ARDS) represents the major cause of mortality [2,3]. A group of COVID-19 patient may have a cytokine storm syndrome, resulting in a rapid failure respiratory function [4]. Multiple guidelines on therapeutic management and support for patients with COVID-19 infection have been developed by several scientific companies [5].

Secondary hemophagocytic lymphohistiocytosis (sHLH) is a hyperinflammatory syndrome secondary to several triggers including sepsis, characterized by a marked increase of cytokines with multi-organ failure and very high mortality rate [6]. sHLH shares with COVID-19 infection an increase in ferritin plasma level and several interleukins (IL): IL-2, IL-6, IL-7, G-CSF, interferon (IFN) gamma, TNF-alpha. Recently, high ferritin and IL-6 plasma values have been associated with lethality rate in COVID-19 infection [3]. Tocilizumab, an IL-6 inhibitor, has been proposed in the treatment of COVID-19 infection.

Ruxolitinib reduces the spleen volume and the circulating values of pro-inflammatory interleukins, in particular IL-6 and TNF-alpha [7]. Recently, preliminary data obtained on 7 sHLH patients treated with ruxolitinib $15 \mathrm{mg}$ bis in die

Giovanni Caocci

giovanni.caocci@unica.it

1 Ematologia e CTMO, Ospedale Businco Azienda Ospedaliera Brotzu, Dipartimento di Scienze Mediche e Sanità Pubblica, Università di Cagliari, Via Jenner, sn, 09124 Cagliari, Italy
(BID) have shown encouraging results on overall survival, observing an improvement of inflammatory markers such as ferritin and the soluble IL-2 receptor [8]. In oncohematological patients, with a variable scenery of immune-depression related to the disease, ruxolitinib has been associated with an increased risk of viral and bacterial infection [9], probably secondary to a cytotoxic T lymphocyte (CTL)-dependent mechanism and a non-cytolytic cytokinedependent mechanism mediated by inflammatory cytokines [10]. Nevertheless, in a patient without oncohematological disease, a short exposition to ruxolitinib will most likely not be sufficient to induce an immune-depleted condition. Since ruxolitinib has proven to be a well-tolerated and relatively manageable drug even in the elderly population with myelofibrosis, it is suggested here to be used in elderly patients with high risk of ARDS secondary to COVID-19 infection.

Eligibility criteria should include patients with age $\geq$ 65 years requiring oxygen treatment with one or more criteria of respiratory function worsening [5]:

(i) Patients has dyspnea or staccato speech (the patient is unable to count rapidly up to 20 after a deep breath) at rest or during minimal activity (sitting up in bed, standing, talking, swallowing, coughing);

(ii) Breathing rate $>22$;

(iii) $\mathrm{PaO}_{2}<65 \mathrm{mmHg}$ or $\mathrm{SpO}_{2}<90 \%$;

(iv) Significant worsening of chest $\mathrm{X}$-ray.

We suggest the following ruxolitinib schedule:

- Dexamethasone $20 \mathrm{mg} / \mathrm{die}$ for 5 day, thereafter $10 \mathrm{mg} / \mathrm{die}$ for 10 days

- Ruxolitinib $15 \mathrm{mg}$ BID for 21 days; in case of clinical worsening and platelets value $>15010^{9} / 1$, increase ruxolitinib until $20 \mathrm{mg}$ BID; after $21 \mathrm{gg}$, whether patient have an improvement, debulk ruxolitinib $10 \mathrm{mg}$ BID for 1 week and $5 \mathrm{mg}$ BID for a further week, before stopping it. 
At baseline and after 5, 10, 20, and 30 days from the beginning of ruxolitinib, all patients should be evaluated for ferritin and serum should be stored for further analysis of IL2, IL6, TNF-alpha, IL-10, and IFN gamma values. Primary endpoints should be considered overall survival at 30 and 60 days, and secondary endpoint should include the cumulative incidence or ARDS onset.

This is a time of a dramatic effort by the scientific community in search of direct anti-viral drugs and supportive therapies aimed to reduce fatal complications of COVID-19 infection.

\section{Compliance with ethical standards}

Conflict of interests The authors declare that they have no conflict of interest.

\section{References}

1. Coronavirus COVID-19 (2019-nCoV) [Internet]. [cited 2020 Mar 29]. Available from: https://gisanddata.maps.arcgis.com/apps/ opsdashboard/index.html\#/bda7594740fd40299423467b48e9ecf6. Accessed 29 Mar 2020

2. He F, Deng Y, Li W (2020) Coronavirus disease 2019 (COVID-19): what we know? J Med Virol. https://doi.org/10.1002/jmv.25766. Online ahead of print. PMID: 32170865

3. Ruan Q, Yang K, Wang W, Jiang L, Song J (2020) Clinical predictors of mortality due to COVID-19 based on an analysis of data of
150 patients from Wuhan, China. Intensive Care Med 46(5):846848

4. Mehta P, McAuley DF, Brown M, Sanchez E, Tattersall RS, Manson; HLH Across Speciality Collaboration (2020) COVID19: consider cytokine storm syndromes and immunosuppression. Lancet Lond Engl 395(10229):1033-1034. https://doi.org/10.1016/ S0140-6736(20)30628-0

5. Simit [Internet]. [cited 2020 Mar 29]. Available from: http://www. simit.org/IT/simit/sezioni-regionali.xhtml/sezione/112-lombardia/ comunicazioni/1. Accessed 29 Mar 2020

6. Seguin A, Galicier L, Boutboul D, Lemiale V, Azoulay E (2016) Pulmonary involvement in patients with hemophagocytic lymphohistiocytosis. Chest. 149(5):1294-1301

7. Arana Yi C, Tam CS, Verstovsek S (2015) Efficacy and safety of ruxolitinib in the treatment of patients with myelofibrosis. Future Oncol Lond Engl 11(5):719-733

8. Ahmed A, Merrill SA, Alsawah F, Bockenstedt P, Campagnaro E, Devata S, Gitlin SD, Kaminski M, Cusick A, Phillips T, Sood S, Talpaz M, Quiery A, Boonstra PS, Wilcox RA (2019 Dec) Ruxolitinib in adult patients with secondary haemophagocytic lymphohistiocytosis: an open-label, single-centre, pilot trial. Lancet Haematol 6(12):e630-e637

9. Sant'Antonio E, Bonifacio M, Breccia M, Rumi E (2019 Nov) A journey through infectious risk associated with ruxolitinib. Br J Haematol 187(3):286-295

10. Caocci G, Murgia F, Podda L, Solinas A, Atzeni S, La Nasa G (2014 Jan) Reactivation of hepatitis B virus infection following ruxolitinib treatment in a patient with myelofibrosis. Leukemia. 28(1):225-227

Publisher's note Springer Nature remains neutral with regard to jurisdictional claims in published maps and institutional affiliations. 